\title{
Occurrence and distribution of fluoride in groundwater of chad formation aquifers in Borno state, Nigeria
}

\begin{abstract}
Borno State is largely underlain by the Chad Formation, which is the youngest stratigraphic sequence in the Chad Basin and most prolific in terms of groundwater resources. The Formation consists of the three prominent water bearing zones known as the Upper, Middle and Lower zone aquifers. The Middle and the Lower zone aquifers are confined while the Upper zone aquifer is unconfined, semi- confined and confined in some places. A total of 175 water samples were collected from boreholes and hand dug wells tapping these aquifers. The samples were collected using plastic bottles and at each sampling point $\mathrm{pH}$, temperature and electrical conductivity were measured using portable $\mathrm{pH} / \mathrm{EC}$ meter. In the laboratory, samples were analysed for fluoride concentration using Spectrophotometer with detection limit of $0.01 \mathrm{mg} / \mathrm{l}$. The Upper zone aquifer has fluoride concentration within the WHO acceptable limit of less than $1.5 \mathrm{mg} / \mathrm{l}$, except for a sample in Magumeri which has concentration of $1.8 \mathrm{mg} / 1$. The Middle and Lower zone aquifers have relatively higher concentrations, with the highest of $4.6 \mathrm{mg} / 1$ in Gubio and $9.00 \mathrm{mg} / 1$ in New Marte respectively. The relatively higher concentrations (above the WHO limit of $1.5 \mathrm{mg} / \mathrm{l}$ ) in these aquifers might be because of leaching from the confining clay layers, enhanced by long residence time and high temperature. The alkaline nature of water in these aquifers and their moderate electrical conductivity favour dissolution of fluoride. In the study area, cases of mild to severe dental fluorosis have been observed in areas with high fluoride concentration - above the WHO limit. In New Marte for instance, where the highest fluoride concentrations was recorded, cases of skeletal fluorosis have been noticed.
\end{abstract}

Volume 2 Issue 4 - 2018

\begin{abstract}
Bura B,' Goni IB, ${ }^{2}$ Sheriff BM, ${ }^{2}$ Gazali $\mathrm{AK}^{3}$
'Chad Basin Development Authority, Maiduguri, Borno State, Nigeria

2PTDF Centre for Basinal Studies, Department of Geology, University of Maiduguri, Nigeria

${ }^{3}$ Department of Geology, University of Maiduguri, Nigeria
\end{abstract}

Correspondence: Goni IB, PTDF Centre for Basinal Studies, Department of Geology, University of Maiduguri, Nigeria, Email ibgoni@yahoo.com

Received: May 14, 2018| Published: August 31, 2018

Keywords: groundwater, aquifer, chad formation, fluoride

\section{Introduction}

The study area lies within latitude $11.15^{\circ}$ and $13.25^{\circ} \mathrm{N}$ and longitude $11.45^{\circ}$ and $14.00^{\circ} \mathrm{E}$ (Figure 1). The area is situated within the semi-arid region of north-eastern Nigeria with an annual rainfall that is generally less than $600 \mathrm{~mm}$ and evapotranspiration of over $2,000 \mathrm{~mm}$. Surface waters are seasonal except for the Lake Chad, therefore, groundwater is the perennial source of water supply for drinking and for other purposes. Groundwater in this part of the State occurs dominantly within the Chad Formation, which is the youngest and the most viable in terms of water resources in the Chad Basin. The Chad Formation comprises of lacustrine sediments that vary in lithology both laterally and vertically. It hosted the three well-defined arenaceous horizons that form the water bearing zones (aquifers). These aquifers are termed the Upper, Middle and the Lower zone aquifers. ${ }^{1}$ Groundwater always contains dissolved and suspended substances of organic and mineral origin. ${ }^{2}$ Therefore, assessing the quality of the water in the study becomes necessary as ingestion of high concentration of some these constituents have health implications. Naturally occurring fluorides in groundwater result from the dissolution of fluoride containing rock minerals, while artificially high fluoride levels occur through contamination from phosphate fertilizers, sewage sludge or pesticide. ${ }^{3}$ Fluoride occurs in three forms, namely, fluorite, apatite or rock phosphate $\left(\mathrm{Ca}_{3} \mathrm{~F}\left(\mathrm{PO}_{4}\right)_{3}\right)$ and cryolite $\left(\mathrm{NaAlF}{ }_{6}\right)$

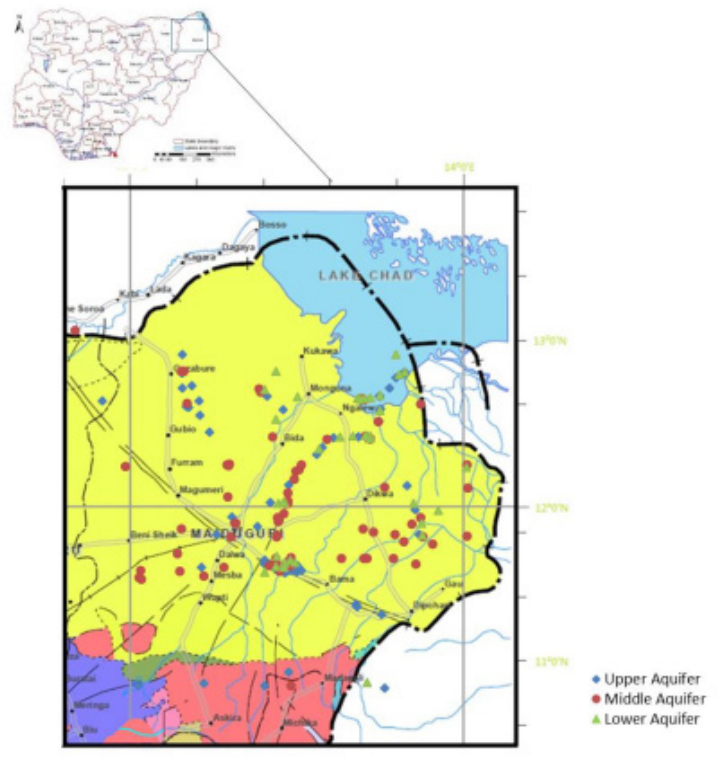

Figure I Map of the study area showing the sampling points.

\section{Geology and hydrogeology of the study area}

The area under study area is located within the Nigerian sector of the Chad Basin and is underlain by the Chad Formation. The geology 
and hydrogeology of the Chad Basin have been described by many authors. ${ }^{1,4-6}$ Various stratigraphic unit were identified in the basin, these include the Bima Sandstone which is the oldest stratigraphic unit (Table 1). The Formation consists of sequence of red sandstone and mudstone of the Continental Group lying unconformably on the basement complex. ${ }^{7}$ The Bima sandstone is overlain by the Gongila Formation and according to Matheis, ${ }^{6}$ the age of the Formation is Cenomanian. The Gongila Formation is overlain by Fika Shale which consists mainly of dark-grey to blue-black shale with occasional gypsum, glauconite and fine-grained sandstone in the upper part of the Formation. ${ }^{4}$ The Gombe Sandstone is of Maastrichtian age and consists of cross-bedded sandstone and siltstone. ${ }^{8}$ The Gombe Sandstone is overlain by the Kerri-Kerri Formation of Palaeocene age and composed of alternating layers of grit and sandstone with

Table I Generalized stratigraphic column of North eastern Nigeria (Modified from Carter et al., ${ }^{4}$ Avbovbo et al. ${ }^{28}$ ) semi-confined to confined.

\begin{tabular}{llll}
\hline Age & Formation & Thickness $(\mathbf{M})$ & Description \\
\hline Quaternary & Chad & 400 & Clay with sand interbeds \\
Palaeocene & Kerri-Kerri & 130 & Predominantly iron-
\end{tabular}

\begin{tabular}{llll}
\hline Uncomformity & Gombe & 315 & $\begin{array}{l}\text { Sandstone, siltstone } \\
\text { and clay beds }\end{array}$ \\
\hline Maastrichtian & & Dark grey to black shale, gypsiferous with limestone beds \\
Senonian & Fika & 430 & Alternating limestone and shale with sand beds \\
Turonian & Gongila & 420 & Poorly sorted, feldspathic sandstone \\
Albian to Cenomanian & Bima & 3050 & Sand/shale succession \\
Albian & Pre-Bima & 800 & \\
\hline Nonconformity & & & \\
\hline Precambrian to Cambrian & Basement Complex & \\
\hline
\end{tabular}

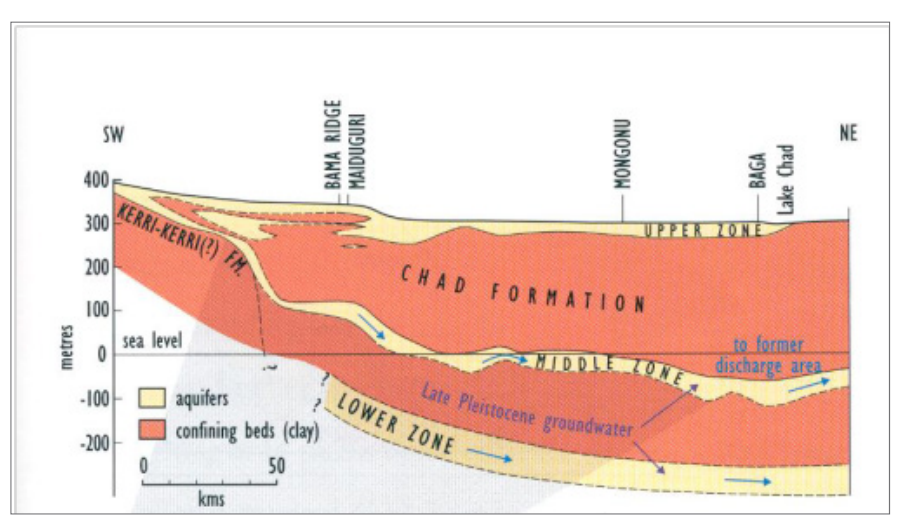

Figure 2 Cross-section of chad formation showing the three aquifer zones (Modified after Miller et $\mathrm{al}^{5}$ ).

The Middle zone aquifer underlies the entire basin, extending to Niger, Chad and Cameroon. ${ }^{10}$ It is separated from the Upper zone aquifer by some thick argillaceous layer. ${ }^{11}$ The aquifer consists of sands, sandy clay and clays with extremely variable proportions in different sections. ${ }^{5}$ The aquifer has shown some considerable lowering of piezometric head..$^{12-14}$ over the last half a centuary. The Lower zone aquifer which was previously known to occur only in Maiduguri area has also been found to be extended up to the fringes of the Lake Chad. ${ }^{12}$ The aquifer is confined, with variable thickness rich sandstone and clay with plinth of laterite

well-developed cross bedding. ${ }^{6}$ The Chad Formation is the youngest stratigraphic unit in the basin (Table 1) and it covers the entire part of the study area. Groundwater resources in the study area are derived from the three aquifers of the Chad Formation. The aquifers are subdivided into Upper, Middle and the Lower zone aquifers on the basis of prominent sandy zones separated by thick clayey members (Figure 2). The Upper zone aquifer contains water under phreatic condition. It is sandy to clayey in composition and attains a thickness of between 5 and 12 meters. It occurs at an average depth of between 40 and 110 meters below ground surface. The aquifer according to Beacon Services and Consulint Intl. Ltd. ${ }^{9}$ is further subdivided into three sub-units known as the $\mathrm{A}, \mathrm{B}$ and $\mathrm{C}$. These units are unconfined,

reaching up to $90 \mathrm{~m}$ in some places..$^{10}$ and occurs at an average depth of 550 to $600 \mathrm{~m}$. The aquifer consists of fine to coarse grained sands and gravels. The age of the groundwater in the Middle and Lower zone aquifers have been estimated at 24 and 18.6 thousand years before present respectively ${ }^{15}$ prior to the last glacial maximum. The mean annual temperature at this recharge time was at least $6^{\circ} \mathrm{C}$ cooler than at the present day based on interpretation of noble gas data.

\section{Methodology}

A total of 175 samples were collected from boreholes and hand dug wells in the three aquifers of the study area. Samples were collected using plastic bottles, which were initially washed with $10 \%$ $\mathrm{HNO} 3$ and rinsed thoroughly with distilled water. The bottles were filled to the brim and sealed by screwing the cap. Samples were stored in frozen box for onward delivery to laboratory. Physical parameters are electrical conductivity, $\mathrm{pH}$ and temperature, which were measured at the sampling point using a portable $\mathrm{pH} / \mathrm{EC}$ meter. Samples were then sent to laboratory for analysis of fluoride concentration using Spectrophotometer (Multiparameter ion specific meter) with a detection limit of $0.001 \mathrm{mg} / \mathrm{l}$. In carrying out the analysis, $4 \mathrm{ml}$ SPADNS reagent was used to prepare a blank. Contour maps and map showing sampling points were constructed using the Surfer 8.0 version computer software. Microsoft excel was also used for linear plots of fluoride concentrations to $\mathrm{pH}$ and EC. 


\section{Results}

The results of fluoride concentration and the physical parameters of samples collected measured in the field for the Upper, Middle and Lower aquifers are presented in Tables 2, Table $3 \&$ Table 4 respectively. The fluoride concentration in the Upper zone aquifer varies between 0.01 and $1.8 \mathrm{mg} / 1$ (Table 2). The highest concentration of $1.8 \mathrm{mg} / \mathrm{l}$ was recorded at the Magumeri Forestry sample. The $\mathrm{pH}$ of the groundwater in the Upper zone aquifer ranges from 6.64 and 8.52 with the highest value obtained at the Kijimatari borehole. The temperature of the water in the Upper zone aquifer ranges from $27.7^{\circ} \mathrm{C}$ to $33.3^{\circ} \mathrm{C}$. There is no wide variation observed in temperature of the Upper zone aquifer. The electrical conductivity in the Upper zone aquifer shows wide variation from 110 to $3,754 \mu \mathrm{S} / \mathrm{cm}$. The electrical conductivity is low around Maiduguri and towards the eastern part (along the Benisheikh axis). It increases tremendously towards the northern part of the study area close to the Lake Chad (Figure 3). The highest conductivity of $3,754 \mu \mathrm{S} / \mathrm{cm}$ was recorded in Cross Kauwa about $50 \mathrm{Km}$ from the Lake Chad (Table 2).

Table 2 Results of fluoride concentrations $(\mathrm{mg} / \mathrm{l})$ and physical parameters of groundwater samples from the Upper zone aquifer

\begin{tabular}{|c|c|c|c|c|c|c|c|c|}
\hline $\begin{array}{l}\text { S/ } \\
\text { no }\end{array}$ & Location & Latitude & Longitude & $\mathrm{pH}$ & $\begin{array}{l}E C(\mu S / \\
\mathrm{cm})\end{array}$ & $\begin{array}{l}\mathrm{T} \\
\left({ }^{\circ} \mathrm{C}\right)\end{array}$ & $\begin{array}{l}F \\
(\mathrm{mg} / \mathrm{l})\end{array}$ & $\begin{array}{l}\text { Depth } \\
\text { (m) }\end{array}$ \\
\hline I & Dikwa West & $12^{0} 02.61^{\prime}$ & $13^{0} 54.36^{\prime}$ & 7.41 & 460 & 31.1 & 0.71 & 56.0 \\
\hline 2 & Dikwa Salleke & $12^{0} 02.13^{\prime}$ & $13^{0} 54.78^{\prime}$ & 7,00 & 293 & 30.0 & 1.26 & 53.0 \\
\hline 3 & Garadai & $12^{0} 14.91$ & $13^{0} 52.91^{\prime}$ & 6.91 & 894 & 30.3 & 0.73 & 49.0 \\
\hline 4 & Umarari II & $11^{0} 52.66$ & $13^{0} 08.04^{\prime \prime}$ & 7.18 & 525 & 31.0 & 0.43 & 61.0 \\
\hline 5 & Low Cost & $11^{0} 51.66$ & $13^{0} 07.44^{\prime \prime}$ & 7.08 & 340 & 31.7 & 0.41 & 72.0 \\
\hline 6 & Polo Road & $11^{0} 08.73$ & $13^{0} 08.73^{\prime \prime}$ & 7.52 & 181 & 29.6 & 0.64 & 94.0 \\
\hline 7 & Circular Road & $11^{0} 48.53^{\prime}$ & $13^{0} 08.81^{\prime}$ & 7 & 208 & 30.8 & 0.59 & 56.0 \\
\hline 8 & 202 estate & $11^{0} 48.12^{\prime}$ & $13^{0} 11.27$ & 7.42 & 255 & 27.7 & 0.30 & 47.0 \\
\hline 9 & Mainok & $11^{0} 49.69^{\prime}$ & $12^{0} 37.53$ & 7 & 161 & 30.8 & 0.58 & 58.0 \\
\hline 10 & Ngamadu & $11^{0} 45.50^{\prime}$ & $12^{0} 15.38^{\prime}$ & 6.66 & 918 & 30.2 & 0.31 & 62.0 \\
\hline II & Mainok & $\left.11^{0} 496\right|^{\prime}$ & $12^{0} 37.98^{\prime}$ & 6.76 & 267 & 31.0 & 0.53 & 53.0 \\
\hline 12 & Damboa Rd & $11^{0} 48.61 "$ & $13^{0} 08.00^{\prime}$ & 7.03 & 185 & 33.3 & 0.01 & 92.0 \\
\hline 13 & Kuluri & $11^{0} 48.39$ & $13^{0} 07.63^{\prime}$ & 7.34 & 186 & 32.4 & 0.49 & 89.0 \\
\hline 14 & Kuluri & $11^{0} 48.34^{\prime}$ & $13^{0} 07.27$ & 6.64 & 110 & 31.9 & 0.57 & 40.0 \\
\hline 15 & Masludemami & $11^{0} 48.04^{\prime}$ & $13^{0} 07.03^{\prime}$ & 6.91 & 145 & 32.3 & 0.45 & 42.0 \\
\hline 16 & Tudun Wada & $11^{0} 50.23$ & $13^{0} 06.68^{\prime}$ & 6.82 & 155 & 31.6 & 0.36 & 65.0 \\
\hline 17 & Dala & $11^{0} 49.42$ & $13^{0} 06.05^{\prime}$ & 6.88 & 169 & 33.1 & 0.45 & 96.0 \\
\hline 18 & Umaranri & $11^{0} 52.52$ & $13^{0} 08.55^{\prime}$ & 7.4 & 550 & 31.8 & 0.00 & 85.0 \\
\hline 19 & Ngarannam & $11^{0} 52.67$ & $13^{0} 08.60^{\prime}$ & 7.38 & 513 & 32.0 & 0.45 & 73.0 \\
\hline 20 & Bulabulin. & $111_{53.18}^{\prime}$ & $13^{0} 08.45^{\prime}$ & 7.23 & 611 & 31.9 & 0.00 & 78.0 \\
\hline 21 & Bulabulin 2 & $11^{0} 53.3 I^{\prime}$ & $13^{0} 09.05^{\prime}$ & 7.27 & 410 & 32.1 & 0.20 & 93.0 \\
\hline 22 & Rail Quarters & $11^{0} 52.46$ & $13^{0} 09.29$ & 7.26 & 511 & 31.6 & 0.00 & 47.0 \\
\hline 23 & Bolori Layout & $11^{0} 51.27$ & $13^{0} 07.70^{\prime}$ & 7.28 & 357 & 2.7 & 0.20 & 56.0 \\
\hline 24 & Bolori Bunu & $11^{0} 51.68^{\prime}$ & $13^{0} 07.88^{\prime}$ & 7.06 & 875 & 32.0 & 0.28 & 59.0 \\
\hline 25 & Bolori Cross & $11^{0} 51.56^{\prime}$ & $13^{0} 07.69^{\prime}$ & 7.03 & 668 & 31.8 & 0.10 & 61.0 \\
\hline 26 & Low Cost II & $11^{0} 51.58$ & $13^{0} 07.32^{\prime}$ & 7.1 & 317 & 33.0 & 0.13 & 68.0 \\
\hline 27 & Kwana Yobe & $11^{0} 50.44^{\prime}$ & $13^{0} 11.47^{\prime}$ & 7.31 & 670 & 32.7 & 0.47 & 72.0 \\
\hline 28 & Jajeri. & $11^{0} 52.25$ & $13^{0} 07.50^{\prime}$ & 7.03 & 405 & 31.5 & 0.34 & 95.0 \\
\hline 29 & Alamderi & $11^{0} 49.09$ & $13^{0} 04.01$ & 6.89 & 215 & 31.6 & 0.25 & 94.0 \\
\hline 30 & GRA & $11^{0} 48.10^{\prime}$ & $13^{0} 08.50^{\prime}$ & 7.63 & 201 & 30.0 & 0.32 & 96.0 \\
\hline 31 & Gambori & $12^{0} 51.97$ & $12^{0} 33.24^{\prime}$ & 8.02 & 382 & 32.6 & 0.91 & 42.0 \\
\hline 32 & Goni bukarli & $12^{0} 49.21$ & $12^{0} 37.22$ & 7.93 & 431 & 31.6 & 0.82 & 48.0 \\
\hline 33 & Mallumti & $12^{0} 42.64$ & $12^{0} 40.34$ & 8.03 & 851 & 32.0 & 0.00 & 56.0 \\
\hline
\end{tabular}


Table continuded..

\begin{tabular}{|c|c|c|c|c|c|c|c|c|}
\hline $\begin{array}{l}\text { S/ } \\
\text { no }\end{array}$ & Location & Latitude & Longitude & $\mathrm{pH}$ & $\begin{array}{l}E C(\mu \mathrm{S} / \\
\mathrm{cm})\end{array}$ & $\begin{array}{l}\text { T } \\
\left({ }^{\circ} \mathrm{C}\right)\end{array}$ & $\begin{array}{l}F \\
(\mathrm{mg} / \mathrm{l})\end{array}$ & $\begin{array}{l}\text { Depth } \\
\text { (m) }\end{array}$ \\
\hline 34 & Zaimolo & $12^{0} 38.70^{\prime}$ & $12^{0} 42.69^{\prime}$ & 7.71 & $|07|$ & 32.2 & 0.32 & 47 \\
\hline 35 & Kareem & $12^{0} 09.57$ & $12^{0} 48.39^{\prime}$ & 7.94 & 217 & 32.0 & 0.38 & NA \\
\hline 36 & Magumeri F & $12^{0} 06.92^{\prime}$ & $12^{0} 48.86^{\prime}$ & 7.64 & $4 I I$ & NA & 1.81 & 96 \\
\hline 37 & Fashar & $12^{0} 06.06^{\prime}$ & $12^{0} 56.94$ & 8.18 & 347 & 31.6 & 0.36 & 83 \\
\hline 38 & Gajiganna & $12^{0} 51.44^{\prime}$ & $13^{0} 06.31^{\prime}$ & 7.04 & 568 & 32.0 & 0.43 & 105 \\
\hline 39 & Ala & $12^{0} 12.41^{\prime}$ & $13^{0} 52.66^{\prime}$ & 7.07 & 756 & 31.1 & 0.11 & 46 \\
\hline 40 & Old marte & $12^{0} 21.68^{\prime}$ & $13^{0} 49.84$ & 8.07 & 953 & 31.7 & 0.35 & 56 \\
\hline 41 & Bama & $11^{0} 31.51^{\prime}$ & $13^{0} 41.16^{\prime}$ & 6.78 & 470 & 30.5 & 1.25 & 95 \\
\hline 42 & Kawuri & $11_{35.41} I^{\prime}$ & $13^{0} 32.12^{\prime}$ & 6.81 & 841 & 29.3 & 1.32 & 76 \\
\hline 43 & Kinjiram & $12^{0} 21.72$ & $13^{0} 08.37^{\prime}$ & 7.6 & 607 & 31.0 & 0.28 & 54 \\
\hline 44 & Burimari & $12^{0} 34.19^{\prime}$ & $13^{0} 18.29^{\prime}$ & 8.14 & 2977 & 31.0 & 0.60 & 49 \\
\hline 45 & Gasarwa & $12^{0} 37.01 '$ & $13^{0} 19.82^{\prime}$ & 7.94 & 3163 & 30.6 & 0.81 & 65 \\
\hline 46 & Aljari & $12^{0} 40.48^{\prime}$ & $13^{0} 23.74$ & 7.88 & 2529 & 31.5 & 0.39 & 48 \\
\hline 47 & Alimatari I & $12^{0} 41.03^{\prime}$ & $13^{0} 33.08^{\prime}$ & 8.52 & 676 & 29.7 & 0.60 & 38 \\
\hline 48 & Kijimatari II & $12^{0} 41.02^{\prime}$ & $13^{0} 33.07$ & 8.39 & 752 & 29.2 & 0.33 & 36 \\
\hline 49 & Mile 90 & $12^{0} 46.72^{\prime}$ & $13^{0} 39.86^{\prime}$ & 7.97 & 1147 & 33.1 & 1.45 & 85 \\
\hline 50 & Dogoshi & $12^{0} 51.67$ & $13^{0} 40.64$ & 8.13 & 3238 & 29.5 & 1.01 & 73 \\
\hline 51 & Cross Kauwa & $12^{0} 56.56^{\prime}$ & $13^{0} 40.37$ & 8.41 & 3511 & 31.8 & 0.40 & 46 \\
\hline 52 & Cross kauwa & $12^{0} 56.53$ & $13^{0} 40.32$ & 8.47 & 3754 & 29.2 & 1.14 & 51 \\
\hline 53 & Garin Giwa & $13^{0} 00.13^{\prime}$ & $13^{0} 43.59^{\prime}$ & 7.84 & 1599 & 33.3 & 0.60 & 40.0 \\
\hline 54 & Baga Military & $13^{0} 03.64^{\prime}$ & $13^{0} 45.73^{\prime}$ & 8.17 & 1269 & 29 & 0.77 & 43.0 \\
\hline 55 & Baga Hausa & $13^{0} 05.8 \mathrm{I}^{\prime}$ & $13^{0} 49.29^{\prime}$ & 8.32 & 1511 & 28.8 & 1.01 & 39.0 \\
\hline 56 & Baga Kasuwa & $13^{0} 06.02^{\prime}$ & $13^{0} 49.16^{\prime}$ & 7.92 & 1726 & 30.2 & 0.66 & 42.0 \\
\hline 57 & Gajagaja I & $13^{0} 05.48^{\prime}$ & $13^{0} 49.35^{\prime}$ & 7.53 & 1690 & & 0.48 & 37.0 \\
\hline 58 & Gajagaja II & $13^{0} 05.46^{\prime}$ & $13^{0} 49.34^{\prime}$ & 7.5 & 1692 & 30.5 & 0.75 & 38.0 \\
\hline 59 & Gajagaja III & $13^{0} 05.44^{\prime}$ & $13^{0} 49.35^{\prime}$ & 7.07 & $135 \mid$ & 31.0 & 0.77 & 39.0 \\
\hline 60 & Gudunbali & $12^{0} 56.57$ & $13^{0} 10.80^{\prime}$ & 7.61 & 1704 & 32.0 & 0.75 & 45.0 \\
\hline 61 & Damasak & $13^{0} 05.93^{\prime}$ & $12^{0} 30.72^{\prime}$ & 7.77 & 236 & 32.0 & 0.33 & 45.0 \\
\hline 62 & Damasak Za & $13^{0} 06.00^{\prime}$ & $12^{0} 30.57^{\prime}$ & 7.46 & 876 & 29.2 & 0.00 & 39.0 \\
\hline 63 & Damasak I & $13^{0} 06.03^{\prime}$ & $12^{0} 30.55^{\prime}$ & 7.31 & 468 & 29.7 & 0.17 & 33.0 \\
\hline 64 & Damasak 2 & $13^{0} 05.95^{\prime}$ & $12^{0} 30.58^{\prime}$ & 7.5 & 233 & 29.7 & 0.25 & 31.0 \\
\hline 65 & Damasak Za & $13^{0} 05.90^{\prime}$ & $12^{0} 30.59^{\prime}$ & 7.5 & 197 & 29.9 & 0.30 & 39.0 \\
\hline 66 & Damasak W & $13^{0} 06.33^{\prime}$ & $12^{0} 30.52$ & 7.31 & 190 & 29.4 & 0.77 & 47.0 \\
\hline 67 & Damasak N & $13^{0} 05.61^{\prime}$ & $12^{0} 31.08^{\prime}$ & 7.09 & 164 & 28.7 & 0.63 & 53.0 \\
\hline 68 & Kinnari & $13^{0} 00.71$ & $12^{0} 33.91$ & 8.01 & 291 & 30.8 & 0.33 & 42.0 \\
\hline 69 & Layi I & $12^{0} 55.05^{\prime}$ & $12^{0} 37.02^{\prime}$ & 8.21 & 676 & 31.0 & 0.69 & 35.0 \\
\hline 70 & Layi II & $12^{0} 55.04$ & $12^{0} 37.10^{\prime}$ & 8.29 & 677 & 30.4 & 0.94 & 33.0 \\
\hline 71 & Gremari & $12^{0} 52.57$ & $12^{0} 31.82^{\prime}$ & 8 & 292 & 30.1 & 0.03 & 46.0 \\
\hline 72 & Bama 2 & $11^{0} 32.30^{\prime}$ & $13^{0} 42.18^{\prime}$ & 6.89 & 512 & 30.3 & 0.85 & 115 \\
\hline 73 & Kawuri & $11^{0} 34.5 I^{\prime}$ & $13^{0} 32.28$ & 6.79 & 831 & 30.1 & 0.65 & 98 \\
\hline 74 & Kawuri I & $11^{0} 34.59^{\circ}$ & $13^{0} 32.39^{\prime}$ & 6.12 & 210 & 30.0 & 0.51 & 89 \\
\hline
\end{tabular}


Table 3 Results of fluoride concentrations $(\mathrm{mg} / \mathrm{l})$ and physical parameters of groundwater samples from the Middle zone aquifer

\begin{tabular}{|c|c|c|c|c|c|c|c|c|}
\hline S/no & Location & Latitude (N) & Long. (E) & $\mathrm{pH}$ & EC $(\mu \mathrm{S} / \mathrm{cm})$ & $\mathrm{T}\left({ }^{\circ} \mathrm{C}\right)$ & $F(m g / l)$ & Depth (m) \\
\hline I & Maiwa & $11^{0} 52.93^{\prime}$ & $13^{0} 27.11$ & 6.78 & 1080 & 36.4 & 0.97 & 303 \\
\hline 2 & Ngarannam & $11^{0} 52.93$ & $13^{0} 34.55^{\prime}$ & 6.66 & 1040 & 36.4 & 0.01 & 318 \\
\hline 3 & Mafa west & $11^{0} 52.93$ & $13^{0} 35.75^{\prime}$ & 6.76 & 790 & 36.4 & 0.25 & 336 \\
\hline 4 & Ajiri yoberi & $11^{0} 52.93^{\prime}$ & $13^{0} 42.50^{\prime}$ & 6.55 & 840 & 36.4 & 1.08 & 296 \\
\hline 5 & Ajiri & $11^{0} 52.93$ & $13^{0} 45.23$ & 6.63 & 869 & 36.4 & 0 & 325 \\
\hline 6 & Farjalari & $11^{0} 58.82$ & $13^{0} 59.11$ & 6.59 & 829 & 39.4 & 0 & 318 \\
\hline 7 & Gajibo & $12^{0} 06.59^{\prime}$ & $13^{0} 51.57^{\prime}$ & 6.75 & 939 & 38.3 & 0 & 322 \\
\hline 8 & N/Marte ISQ & $12^{0} 15.09^{\prime}$ & $13^{0} 08.09^{\prime}$ & 7.61 & 581 & 37.3 & 1.4 & 380 \\
\hline 9 & Umarari & $11^{0} 52.46$ & $13^{0} 09.05^{\prime}$ & 6.8 & 408 & 38.3 & 1.7 & 273 \\
\hline 10 & Hausari & $11^{0} 50.39$ & $13^{0} 09.08$ & 7.36 & 190 & 36 & 0.67 & 264 \\
\hline II & Hausari Goro & $11^{0} 50.48^{\prime}$ & $13^{0} 00.99^{\prime}$ & 6.8 & 390 & 36.4 & 0.29 & 258 \\
\hline 12 & Njimtilo & $11^{0} 54.91^{\prime}$ & $12^{0} 29.27$ & 6.87 & 630 & 37 & 0.95 & 279 \\
\hline 13 & Benisheikh & $1 \mathrm{I}^{0} 48.44^{\prime}$ & $12^{0} 29.68^{\prime}$ & 6.87 & 233 & 35.3 & 0.08 & 262 \\
\hline 14 & Benisheikh K & $11^{0} 48.63$ & $12^{0} 15.76^{\prime}$ & 7.1 & 253 & 35.5 & 0.56 & 253 \\
\hline 15 & Ngamdu Alh & $11^{0} 45.67$ & $12^{0} 15.45^{\prime}$ & 7.35 & 225 & 36.6 & 0.19 & 252 \\
\hline 16 & Ngamdu & $11^{0} 45.26^{\prime}$ & $12^{0} 16.45^{\prime}$ & 7.91 & 467 & 35.8 & 0.52 & 264 \\
\hline 17 & TC Ngamdu & $11^{0} 46.42^{\prime}$ & $12^{0} 37.96$ & 7.25 & 323 & 36 & 0.61 & 257 \\
\hline 18 & Mainok East & $1 \mathrm{I}^{0} 49.78$ & $12^{0} 45.5 \mid$ & 7.3 & 202 & 35.4 & 0.41 & 253 \\
\hline 19 & Jakana West & $11^{0} 50.26^{\prime}$ & $13^{0} 07.74^{\prime}$ & 6.32 & 555 & 36.2 & 0 & 215 \\
\hline 20 & Shuwari & $11^{0} 48.35$ & $13^{0} 04.91$ & 7.1 & 158 & 36.3 & 0.68 & 282 \\
\hline 21 & Ngomari & $11^{0} 49.87$ & $13^{0} 04.82$ & 7.59 & 218 & 35.2 & 0 & 278 \\
\hline 22 & Ngomari Bus Stop & $11^{0} 49.50^{\prime}$ & $13^{0} 08.87$ & 7.16 & 186 & 35.1 & 0.08 & 269 \\
\hline 23 & Bulabulin & $11^{0} 53.52$ & $13^{0} 08.92^{\prime}$ & 7.18 & 440 & 38.2 & 0.94 & 253 \\
\hline 24 & Umararil & $11^{0} 51.17$ & $13^{0} 52.76^{\prime}$ & 6.85 & 415 & 37.9 & 0.3 & 263 \\
\hline 25 & Ala & $12^{0} 12.87$ & $\mathrm{I}^{0} 303.69$ & 7.11 & 588 & 36.7 & 1.1 & 344 \\
\hline 26 & Tungusher & $12^{0} 01.78^{\prime}$ & $14^{0} 10.77^{\prime}$ & 7.21 & 650 & 36.1 & 0.06 & 285 \\
\hline 27 & Ngala CBDA & $12^{0} 20.84^{\prime}$ & $13^{0} 41.76^{\prime}$ & 8.06 & 693 & 40.2 & 3.2 & 314 \\
\hline 28 & Bundur & $12^{0} 53.47^{\prime}$ & $13^{0} 54.66^{\prime}$ & 7.3 & 477 & $4 I .1$ & 0.86 & 390 \\
\hline 29 & Ala & $12^{0} 08.93$ & $13^{0} 04.44^{\prime}$ & 6.66 & 950 & 38 & 0.35 & 344 \\
\hline 30 & Suwurmari & $12^{0} 07.65^{\prime}$ & $13^{0} 04.45^{\prime}$ & 7.03 & 586 & 40.1 & 1.74 & 360 \\
\hline 31 & Karnowa & $12^{0} 08.35^{\prime}$ & $13^{0} 04.67^{\prime}$ & 7.11 & 581 & 39.5 & 0.57 & 345 \\
\hline 32 & Wulomari & $12^{0} 10.64$ & $13^{0} 06.57$ & 7.1 & 601 & 38.9 & 0.22 & 328 \\
\hline 33 & Gajiganna & $12^{0} 15.66^{\prime}$ & $13^{0} 07.81^{\prime}$ & 7.2 & 615 & 40.1 & 0.3 & 341 \\
\hline 34 & Zindur & $12^{0} 18.76^{\prime}$ & $13^{0} 07.81$ & 7.17 & 576 & 40.2 & 0.73 & 354 \\
\hline 35 & Kulu Kawiya & $12^{0} 24.33^{\prime}$ & $13^{0} 09.95^{\prime}$ & 7.66 & 616 & 39.2 & 0.47 & 315 \\
\hline 36 & Kote & $12^{0} 26.96^{\prime}$ & $13^{0} 11.12^{\prime}$ & 7.37 & 575 & 38.7 & 0.38 & 296 \\
\hline 37 & Kalaa & $12^{0} 27.69$ & $13^{0} 11.67$ & 7.08 & 545 & 40.2 & 0.27 & 325 \\
\hline 38 & Gajiram & $12^{0} 29.69$ & $13^{0} 12.71$ & 7.67 & 541 & 39.6 & 0.32 & 342 \\
\hline 39 & Ali Gambori & $12^{0} 39.59^{\prime}$ & $13^{0} 22.13^{\prime}$ & 7.31 & 406 & 39.8 & 0.5 & 336 \\
\hline 40 & Garu kimel & $12^{0} 41.14^{\prime}$ & $13^{0} 34.97$ & 7.37 & 440 & 39.9 & 0.43 & 358 \\
\hline
\end{tabular}


Table continuded...

\begin{tabular}{|c|c|c|c|c|c|c|c|c|}
\hline S/no & Location & Latitude (N) & Long. (E) & $\mathrm{pH}$ & $\mathrm{EC}(\mu \mathrm{S} / \mathrm{cm})$ & $\mathrm{T}\left({ }^{\circ} \mathrm{C}\right)$ & $F(\mathrm{mg} / \mathrm{l})$ & Depth (m) \\
\hline 41 & Mongun Est & $12^{0} 41.03^{\prime}$ & $13^{0} 36.50^{\prime}$ & 7.31 & 427 & 39.4 & 0.61 & 362 \\
\hline 42 & Monguno & $12^{0} 40.07$ & $13^{0} 37.16$ & 7.28 & 386 & 38.2 & 0.39 & 358 \\
\hline 43 & Monguno S & $12^{0} 39.89^{\prime}$ & $13^{0} 37.17^{\prime}$ & 7.26 & 433 & 38.8 & 1.17 & 360 \\
\hline 44 & Monguno C & $12^{0} 40.47^{\prime}$ & $13^{0} 36.35^{\prime}$ & 7.33 & 415 & 39.9 & 0.54 & 373 \\
\hline 45 & Mile 90 & $12^{0} 46.66^{\prime}$ & $13^{0} 39.74$ & 7.31 & 372 & 41.6 & 1.39 & 348 \\
\hline 46 & Yoyo & $12^{0} 42.80^{\prime}$ & $13^{0} 38.24$ & 731 & 378 & 39.6 & 1.19 & 311 \\
\hline 47 & Baga tasha & $12^{0} 05.60^{\prime}$ & $13^{0} 49.13^{\prime}$ & 7.1 & 1337 & 39.2 & 0.68 & 451 \\
\hline 48 & Kukawa I & I2v55.75 & $13^{0} 34.54$ & 7.27 & 734 & 39.1 & 0 & 325 \\
\hline 49 & Kukawa 2 & $12^{0} 55.16^{\prime}$ & $13^{0} 34.63^{\prime}$ & 7.12 & 611 & 39.3 & 0.13 & 318 \\
\hline 50 & Kukawa 3 & $12^{0} 55.55$ & $13^{0} 33.77^{\prime}$ & 7.35 & 382 & 40.3 & 1.58 & 299 \\
\hline 51 & Kukawa 4 & $12^{0} 55.43$ & $13^{0} 33.61$ & 7.25 & 567 & 35.8 & 0.42 & 321 \\
\hline 52 & Damasak I & $13^{0} 05.94$ & $12^{0} 30.92^{\prime}$ & 7.73 & 184 & 36.1 & 0.35 & 325 \\
\hline 53 & Damasak 2 & $13^{0} 06.37^{\prime}$ & $12^{0} 30.46^{\prime}$ & 7.6 & 160 & 39.6 & 0 & 333 \\
\hline 54 & Wakilti & $12^{0} 53.69^{\prime}$ & $12^{0} 32.53^{\prime}$ & 6.87 & 1166 & 37.3 & 0.78 & 321 \\
\hline 55 & Kareto & $12^{0} 53.30^{\prime}$ & $12^{0} 30.48^{\prime}$ & 7.21 & 1177 & 37.5 & 0.86 & 311 \\
\hline 56 & Gubio park & $12^{0} 29.72^{\prime}$ & $12^{0} 46.54^{\prime}$ & 7.67 & 256 & 38.2 & 1.75 & 339 \\
\hline 57 & Gubio kumbu & $12^{0} 30.12^{\prime}$ & $12^{0} 46.81^{\prime}$ & 7.45 & 257 & 38.1 & 1.7 & 341 \\
\hline 58 & Gubio Central & $12^{0} 29.89^{\prime}$ & $12^{0} 46.99^{\prime}$ & 7.43 & 256 & 39.1 & 1.5 & 344 \\
\hline 59 & Gubio Cross & $12^{0} 29.58^{\prime}$ & $12^{0} 47.17^{\prime}$ & 7.56 & 255 & 37.2 & 4.6 & 325 \\
\hline 60 & Golaram & $12^{0} 21.80^{\prime}$ & $12^{0} 47.78^{\prime}$ & 7.36 & 394 & 38.4 & 0.64 & 346 \\
\hline 61 & Madamari & $12^{0} 17.33^{\prime}$ & $12^{0} 47.08^{\prime}$ & 7.73 & 294 & 38.9 & 1.35 & 327 \\
\hline 62 & Magumeril & $12^{0} 06.87^{\prime}$ & $12^{0} 49.43^{\prime}$ & 7.55 & 402 & 39.2 & 1.9 & 339 \\
\hline 63 & Magumeri 2 & $12^{0} 06.85^{\prime}$ & $12^{0} 49.57^{\prime}$ & 7.51 & 339 & 37.8 & 4.5 & 354 \\
\hline 64 & Goniri & $12^{0} 59.49^{\prime}$ & $12^{0} 57.63^{\prime}$ & 7.93 & 430 & 38 & 0.94 & 298 \\
\hline 65 & Hoyo & $12^{0} 58.4 I^{\prime}$ & $12^{0} 58.47$ & 8.31 & 435 & 37.5 & 0.34 & 313 \\
\hline 66 & Wulgo & $12^{0} 29.32^{\prime}$ & $12^{0} 10.67^{\prime}$ & 6.85 & 658 & 38.6 & 0.48 & 341 \\
\hline 67 & Kaje & $12^{0} 08.94^{\prime}$ & $13^{0} 54.66$ & 6.86 & 812 & 39.2 & 0.48 & 334 \\
\hline 68 & Wulgo2 & $12^{0} 29.65^{\prime}$ & $14^{0} 10.85^{\prime}$ & 6.93 & 739 & 38.8 & 0.56 & 325 \\
\hline 69 & Limanti & $13^{0} 22.35^{\prime}$ & $11^{0} 52.84$ & 6.85 & 825 & 37.9 & 0.25 & 308 \\
\hline 70 & Kirenowa & $12^{0} 25.70^{\prime}$ & $13^{0} 55.57$ & 6.83 & 612 & 38.7 & 0.62 & 296 \\
\hline 71 & Tungushe & $12^{0} 01.65^{\prime}$ & $13^{0} 03.72^{\prime}$ & 6.8 & 637 & 36.1 & 0.33 & 285 \\
\hline 72 & Ngala East & $12^{0} 21.05^{\prime}$ & $14^{0} 10.93$ & 8.01 & 701 & 39.5 & 1.95 & 321 \\
\hline 73 & Tungushe I & $12^{0} 02.33^{\prime}$ & $13^{0} 04.32^{\prime}$ & 6.78 & 801 & 36.7 & 0.05 & 264 \\
\hline 74 & Baale & $12^{0} 03.00^{\prime}$ & $13^{0} 46.00^{\prime}$ & 7.23 & 573 & 37.1 & 0.15 & 307 \\
\hline
\end{tabular}

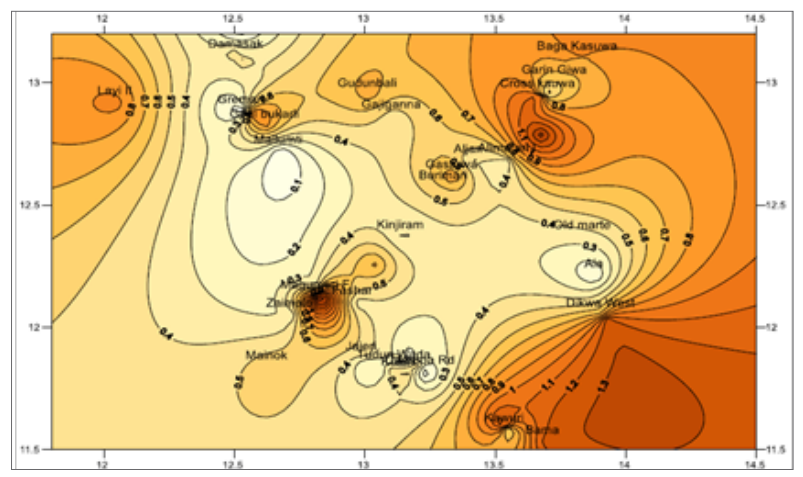

Figure 3 Distribution of fluoride in the upper aquifer groundwater of the chad formation. 
Table 4 Results of fluoride concentrations $(\mathrm{mg} / \mathrm{l})$ and physical parameters of groundwater samples from the Lower zone aquifer

\begin{tabular}{|c|c|c|c|c|c|c|c|c|}
\hline S/no & Location & Latitude (N) & Longitude (E) & $\mathrm{pH}$ & $\mathrm{EC}(\mu \mathrm{S} / \mathrm{cm})$ & $\mathrm{T}\left({ }^{\circ} \mathrm{C}\right)$ & $F(\mathrm{mg} / \mathrm{l})$ & Depth (m) \\
\hline 1 & Mafa Hausari & $110_{55.15}$ & $13^{0} 36.15^{\prime}$ & 7.04 & 573 & 44.4 & 1.42 & 575 \\
\hline 2 & Dikwa Ajari & $12^{0} 01.92$ & $13^{0} 55.38$ & 6.75 & 791 & 43.3 & 1.05 & 563 \\
\hline 3 & Logomani & $12^{0} 12.11$ & $14^{0} 01.08$ & 6.83 & 817 & 44.5 & 0.32 & 590 \\
\hline 4 & Albanya & $12^{0} 07.18^{\prime}$ & $13^{0} 54.85$ & 6.84 & 870 & $4 I .4$ & 0.54 & 597 \\
\hline 5 & N/Marte ISQ & $12^{0} 14.72$ & $13^{0} 51.97$ & 8.00 & 623 & 43.2 & 9.00 & 589 \\
\hline 6 & N/MarteSSQ & $12^{0} 15.04$ & $13^{0} 51.90^{\prime}$ & 7.99 & 522 & 44.5 & 6.00 & 593 \\
\hline 7 & Pompomari & $11^{0} 51.44$ & $13^{0} 06.59$ & 7.52 & 304 & 41.6 & 2.26 & 612 \\
\hline 8 & Bolori & $11^{0} 51.63$ & $13^{0} 06.63^{\prime}$ & 7.48 & 320 & 50.6 & 3.30 & 618 \\
\hline 9 & Bulumkutu & $11^{0} 50.05$ & $13^{0} 06.51$ & 7.45 & 282 & 43.6 & 0.77 & 598 \\
\hline 10 & Ngarannam PTF & $11^{0} 52.67$ & $13^{0} 09.05$ & 7.48 & 334 & 49.8 & 3.15 & 618 \\
\hline II & Old Maidugu & $11^{0} 51.69$ & $13^{0} 10.43^{\prime}$ & 7.34 & 276 & 42.6 & 0.63 & 594 \\
\hline 12 & 777 Estate & $11^{0} 49.83$ & $13^{0} 03.58$ & 7.53 & 325 & 42.1 & I.II & 468 \\
\hline 13 & Kekeno & $12^{0} 47.46^{\prime}$ & $13^{0} 40.30^{\prime}$ & 7.36 & 380 & 41.2 & 0.89 & 563 \\
\hline 14 & Suleimanti $F$ & $11^{0} 47.84$ & $13^{0} 07.80$ & 7.49 & 273 & 40.8 & 0.60 & 588 \\
\hline 15 & Cross kauwa & $12^{0} 56.75^{\prime}$ & $13^{0} 40.38^{\prime}$ & 7.13 & 700 & 42.9 & 1.13 & 577 \\
\hline 16 & Gajiganna NNPC & $12^{0} 15.19^{\prime}$ & $13^{0} 07.11$ & 6.91 & 894 & 41.5 & 0.40 & 610 \\
\hline 17 & Gajiganna & $12^{0} 14.85^{\prime}$ & $13^{0} 06.32$ & 7.76 & 393 & 45.7 & 2.10 & 596 \\
\hline 18 & Jigalta & $12^{0} 36.31$ & $13^{0} 19.17$ & 7.16 & 412 & 40.6 & 0.43 & 578 \\
\hline 19 & Baga CBDA & $13^{0} 04.00$ & $13^{0} 46.25^{\prime}$ & 7.18 & 1050 & 42.9 & 0.30 & 524 \\
\hline 20 & Baga Fishery & $13^{0} 04.79^{\prime}$ & $13^{0} 46.92$ & 6.98 & 1418 & $4 I .1$ & 0.19 & 563 \\
\hline 21 & Baga Bud & $13^{0} 05.95$ & $13^{0} 49.80$ & 6.95 & 1040 & 42.5 & 0.69 & 571 \\
\hline 22 & Kukawa Gwange & $12^{0} 55.88^{\prime}$ & $13^{0} 36.77$ & 7.18 & 576 & 42.1 & 0.08 & 523 \\
\hline 23 & Mile IKukaw & $12^{0} 55.59^{\prime}$ & $13^{0} 35.17$ & 7.30 & 360 & 43.7 & 2.90 & 585 \\
\hline 24 & Lawsa Filling Station & $11^{0} 54.29$ & $13^{0} 04.25^{\prime}$ & 8.22 & 312 & 43 & 3.80 & 611 \\
\hline 25 & Monguno Irrigation & $12^{0} 41.22$ & $13^{0} 37.23^{\prime}$ & 7.28 & 363 & 40.9 & 0.88 & 566 \\
\hline 26 & Madari & $12^{0} 50.75^{\prime}$ & $13^{0} 39.94$ & 7.3 & 436 & 40.9 & 0.19 & 590 \\
\hline 27 & Mairari & $12^{0} 41.02$ & $13^{0} 26.33^{\prime}$ & 7.36 & 422 & 40.1 & 4.60 & 588 \\
\hline 28 & Lingir & $12^{0} 41.29^{\prime}$ & $13^{0} 30.59$ & 7.23 & 392 & 40.3 & 0.83 & 596 \\
\hline 29 & Mongunu Forestry & $12^{0} 41.23$ & $13^{0} 35.87$ & 7.32 & 408 & 41.3 & 0.97 & 578 \\
\hline 30 & Monguno BH5 & $12^{0} 39.81$ & $13^{0} 36.62^{\prime}$ & 7.35 & 401 & 40.5 & 0.97 & 568 \\
\hline 31 & Baga Mission & $\left.13^{0} 05.7\right|^{\prime}$ & $13^{0} 48.86$ & 6.95 & 1295 & 41.4 & 0.04 & 563 \\
\hline 32 & Sijjin & $12^{0} 55.67$ & $13^{0} 32.42^{\prime}$ & 7.24 & 398 & 43 & 0.85 & 578 \\
\hline 33 & Kukawa Garage & $12^{0} 55.59$ & $13^{0} 33.58$ & 7.32 & 381 & 42 & 0.26 & 593 \\
\hline 34 & Gudumbali kagunri & $12^{0} 56.34$ & $13^{0} 10.98^{\prime}$ & 6.97 & 959 & 43.1 & 1.11 & 603 \\
\hline 35 & Kukawa View Center & $12^{0} 55.47$ & $13^{0} 34.01$ & 7.08 & 723 & 40.5 & 0.67 & 611 \\
\hline 36 & Garu Kimel & $12^{0} 40.98$ & $13^{0} 34.95$ & 7.63 & 374 & 40.3 & 0.87 & 589 \\
\hline 37 & Wulgo & $12^{0} 29.15$ & $14^{0} 10.65$ & 6.61 & 522 & 41.8 & 0.67 & 593 \\
\hline 38 & Granda & $12^{0} 59.21$ & $13^{0} 00.05$ & 6.51 & 725 & 40.7 & 1.25 & 573 \\
\hline
\end{tabular}


In the Middle zone aquifer, the concentration of fluoride ranges from 0.01 and $4.6 \mathrm{mg} / \mathrm{l}$. Nine samples out of the 74 analysed for this aquifer show fluoride concentrations above the $\mathrm{WHO}^{16}$ permissible limit of $1.5 \mathrm{mg} / 1$. About $75 \%$ of the samples have fluoride concentration below the permissible limit. Four samples collected within Gubio Town recoded fluoride concentration above $1.5 \mathrm{mg} / 1$ (Table 3). One well located at Ngala the north-eastern part of the study area showed fluoride concentration up to $3.2 \mathrm{mg} / 1$. Figure 4 shows the distribution of fluoride in the Middle zone aquifer across the study area. The $\mathrm{pH}$ values of groundwater in the Middle zone aquifer vary from 6.32 to 8.31 , with over $70 \%$ of the samples having $\mathrm{pH}$ values of greater than 7.00. The highest temperature recorded for the Middle zone aquifer groundwater is $41.3^{\circ} \mathrm{C}$ at Mile 90 . Over $80 \%$ of the samples from this aquifer have groundwater temperature greater than $36^{\circ} \mathrm{C}$. The electrical conductivity of groundwater in this zone varies from 158 to $1,337 \mu \mathrm{S} / \mathrm{cm}$. The conductivity is low around Maiduguri just like the Upper zone but further north around Kareto axis (Figure 4) it is as high as $1,166 \mu \mathrm{S} / \mathrm{cm}$.

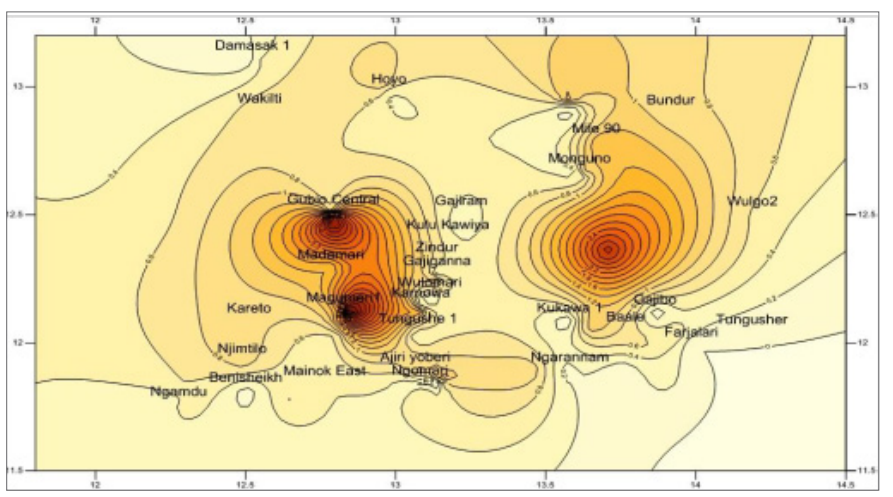

Figure 4 Distribution of fluoride in the middle aquifer groundwater of the chad formation.

The Lower zone aquifer recorded the highest concentration of fluoride of about $9.00 \mathrm{mg} / 1$ at New Marte and a second well in the town equally recorded high fluoride concentrations of $6.00 \mathrm{mg} / \mathrm{l}$ (Table 4). The corresponding temperatures of these wells are as high as $44.5^{\circ} \mathrm{C} 43.2^{\circ} \mathrm{C}$ and the values of the $\mathrm{pH}$ are 8.00 and 7.99 respectively. The high fluoride concentrations are widely distributed across the study area. The fluoride level within Maiduguri, for instances in places like Bolori, Ngarranam, Pompommari, recorded about 3.30, $2.26,3.15,3.80 \mathrm{mg} / 1$ respectively. The maximum temperature of $50.6^{\circ} \mathrm{C}$ was recorded at Bolori in Maiduguri, the southern part of the study area (Figure 5). The lowest temperature $\left(40.1^{\circ} \mathrm{C}\right)$ in the zone was obtained at Mairari Village which recorded fluoride concentration of about $5.0 \mathrm{mg} / 1$. The lowest electrical conductivity $(273 \mu \mathrm{S} / \mathrm{cm})$ in the Lower zone was also obtained at Maiduguri and the maximum value of about $1,418 \mu \mathrm{S} / \mathrm{cm}$ was obtained at Baga extreme northeastern part of the study area. This shows that the distribution of conductivity in all the three aquifers show similar trend; that is, increases towards the northern and NE parts of the study area, close to the Lake Chad. Contour of fluoride concentrations in groundwater from the three aquifers of the study area are plotted in Figure 3, Figure 4 \& Figure 5 to show their spatial distribution. A linear plot of electrical conductivity against the concentrations of fluoride for the samples of the study area is carried out in Figure 6 to evaluate their relationship. Figure 7 is a plot of fluoride concentrations to groundwater sample depth from the three aquifers of the Chad Formation. This is to evaluate their depth relationship.

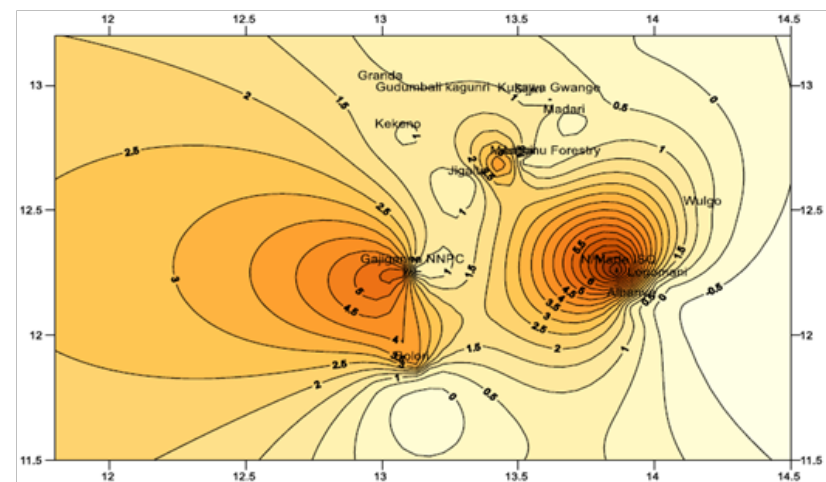

Figure 5 Distribution of fluoride in the lower aquifer groundwater of the chad formation.

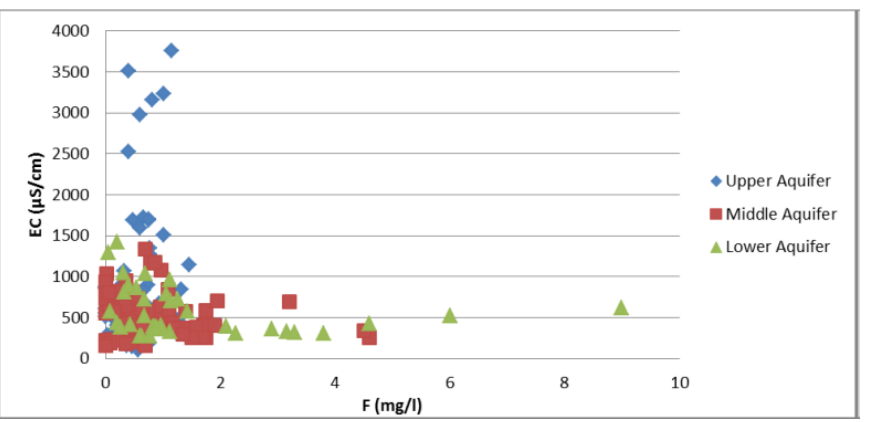

Figure 6 Plot of fluoride versus electrical conductivity (EC) for the chad formation aquifers.

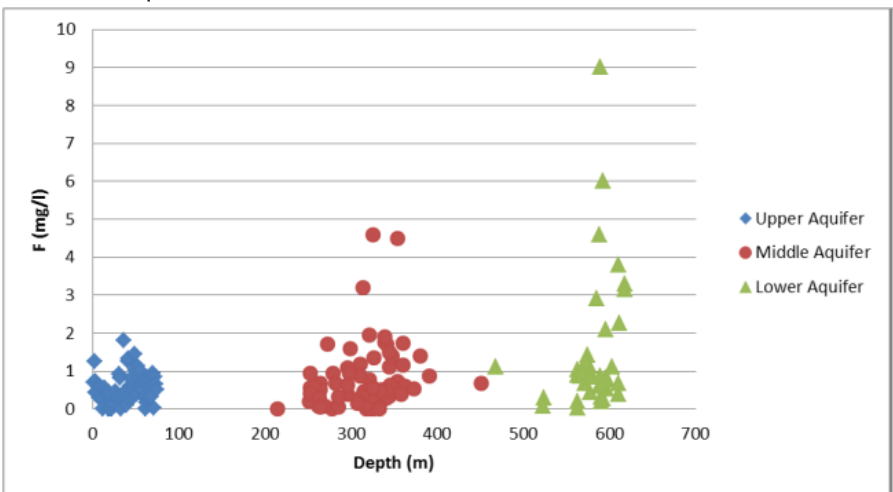

Figure 7 Plot of flouride concentrations to groundwater sample depth for the chad formation aquifers.

\section{Discussion}

The occurrence and distribution of fluoride in the Upper, Middle and Lower zone aquifer across the study area are shown in Figure 3 , Figure $4 \&$ Figure 5 respectively. The fluoride contour map for the Upper zone aquifer (Figure 3) shows a low fluoride concentration (less than $1.5 \mathrm{mg} / \mathrm{l}$ ) for most parts, except for a sample at Monguno that is $1.8 \mathrm{mg} / \mathrm{l}$, which is slightly above the $\mathrm{WHO}^{16}$ permissible limit of $1.5 \mathrm{mg} / 1$. The low concentration in the Upper zone aquifer could probably be attributed to the shallow phreatic nature of the aquifer with active groundwater recharge. Map of fluoride concentrations to depth (Figure 7) shows that high fluoride concentrations are found in the deeper confined aquifer. The highest concentration of $9 \mathrm{mg} / 1$ was recorded in a well tapping the Lower zone aquifer at a depth of $589 \mathrm{~m}$. The Middle zone aquifer at a depth of between $250 \mathrm{~m}$ to $400 \mathrm{~m}$, also shows high fluoride concentrations, with the highest of 
$4.6 \mathrm{mg} / \mathrm{l}$ at the Gubio Cross borehole. This is consistent with the work of Vasquez et al., ${ }^{17}$ that water with higher fluoride content comes from deep aquifers. Vasquez et al., ${ }^{17}$ have also mentioned that increased fluoride concentrations is related to longer residence time of water and the deeper Middle and Lower aquifers of the study area do have palaeowaters, ${ }^{18}$ recharged between 24,000 and 18,400 years before present. ${ }^{15}$ Similar conclusions were reached by Ramakrishnan ${ }^{19}$ who added that the longer residence time will give longer reactions time with the aquifer materials and if they contain fluoride will lead to its dissolution.

Leakage of fluoride rich pore fluid from intervening clay rich aquitard layers induced by excessive exploitation of groundwater is also a potential source of the fluoride. ${ }^{20}$ In the study area, both the Middle and Lower zone aquifers are confined by thick (about $100 \mathrm{~m}$ ) clay layers. Also, in the case of the Middle zone aquifer decline in its piezometric head of over $15 \mathrm{~m}$ in the last 50 years has been reported. ${ }^{14}$ which they attribute to over exploitation. This may explain the source of high fluoride in these aquifers in the line with the position of Gupta. ${ }^{20}$ From the foregone, therefore, contact time and leaching from fluoride bearing minerals within the clays may be important factors controlling the occurrence and distribution of fluoride in the study area. The low fluoride concentrations in the Upper aquifer is because of lack of the confining clay layer. Linear plot of fluoride to electrical conductivity (Figure 6) did not show a clear relationship or trend, which indicates that fluoride concentrations does not control the value of the electrical conductivity. The same trend is observed between the $\mathrm{pH}$ values and fluoride concentrations. Although, at the boreholes in New Marte where both samples recorded high fluoride concentrations of 9.00 and $6.00 \mathrm{mg} / 1$, their $\mathrm{pH}$ values are 8.00 and 7.99 respectively, while their electrical conductivity values are moderate at 623 and $522 \mu \mathrm{S} / \mathrm{cm}$ respectively. This is similar to the conclusions of Saxena \& Ahmed ${ }^{21}$ that alkaline $\mathrm{pH}$ (7.6 and 8.6) and moderate electrical conductivity are favourable conditions for dissolution of fluoride bearing minerals. The relatively high temperature of the deeper zones also enhances the dissolution. Chandrasekharam \& $\mathrm{Antu}^{22}$ have mentioned that water rock interaction is enhanced by elevated temperature. In the study area, the alkaline nature and high temperature of the groundwater in the confined aquifers (Middle and Lower) may have favour the dissolution of fluoride and thus explain its high concentrations in these aquifers.

High fluoride concentrations in groundwater has also been linked to high evapotranspiration in arid regions. ${ }^{23}$ Although, the study area also falls within the semi-arid and arid region with high evapotranspiration rate, this link has not been observed. Because the aquifer that could have been affected most will be the Upper aquifer in this area, but it is the one with low fluoride concentrations. Normal fluoride content in atmospheric air is reported to be between $0.01-0.4 \mathrm{mg} / 1^{24}$ Average fluoride content of precipitation varies from almost zero to $0.089 \mathrm{mg} / 1 .{ }^{25}$ This may contribute some fluoride to the Upper zone aquifer, as it receives present day recharged from rainfall. Fluoride is an indispensable element for the maintenance of dental health. ${ }^{17}$ It is well known that the excess fluoride intake is responsible for dental and skeletal fluorosis. ${ }^{26}$ The cases of dental fluorosis have been observed in areas where fluoride concentration is greater than $1.5 \mathrm{mg} / \mathrm{l}$. These cases are rampant among children living in the area. The dental fluorosis seen in the study area is mostly characterised by brown to black staining on the surface of the teeth. These types were seen at Bolori and Pompommari where the fluoride concentration reaches up to 3.30 and $2.26 \mathrm{mg} / 1$ respectively. Although, the extent of the damage differs from one individual to the other, perhaps due to levels of exposure and varying nutritional status of individual. ${ }^{27}$ In Gubio area where the two boreholes have fluoride concentrations of $4.6 \mathrm{mg} / 1$ and $1.7 \mathrm{mg} / \mathrm{l}$, the level of dental fluorosis observed is severe with eroded teeth and some have lost their shapes and sizes. Skeletal fluorosis has been seen in New Marte where the two boreholes have fluoride concentrations of $9 \mathrm{mg} / 1$ and $6.00 \mathrm{mg} / 1 .^{28}$

\section{Conclusion}

The occurrence and distribution of fluoride in groundwater of Chad Formation aquifers in Borno State show that fluoride concentrations vary widely, but generally higher in the confined Middle and Lower zone aquifers. The low fluoride concentrations in the Upper aquifer could probably be attributed to the shallow phreatic nature of the aquifer with active groundwater recharge. The high fluoride concentrations in Middle and Lower aquifers result from its dissolution from fluoride bearing minerals probably within the confining clay horizons. This is enhanced by the long residence time of water and high temperature in these aquifers facilitating water-rock interaction. The alkaline nature of water in these aquifers and their moderate electrical conductivity are also favourable conditions for fluoride dissolution. Fluoride concentrations above the WHO permissible limit of $1.5 \mathrm{mg} / \mathrm{l}$ have been recorded in many places in the study area. In these parts, cases of dental fluorosis have been observed manifested by black staining on the surface of the teeth. In locations where fluoride concentrations are very high eroded teeth and lost in shape and size have been observed. In New Marte town where the concentrations reached $9 \mathrm{mg} / 1$ cases of skeletal fluorosis have been seen.

\section{Acknowledgement}

None.

\section{Conflict of interest}

The authors declare there is no conflict of interest.

\section{References}

1. Barber W, Jones. The geology and hydrogeology, Bornu province. Record Geological survey of Nigeria. 1960.

2. Jain P, Sharam JD, Sohu D, et al. chemical analysis of drinking water of cillages of Sanganer Teshil Jaipur District. Journ of $n$ Enuiron Science and Tech. 2005;2(4):373-375.

3. EPA. Public health global for fluoride in drinking water. Pesticide and environmental toxicology. California environmental protection Agency. 1997.

4. Carter JD, Barber W, Tait EA, et al. The geology of parts of Adamawa, Bauchi and Borno provinces in North-eastern Nigeria. Bull Geol Surv NIg. 1963;30:109-109.

5. Miller RE, Johnson RH, Oluwu JAI, et al. Availability of groundwater in the Chad Basin of Bornu and Dikwa Emirates, Northern Nigeria. U.S. Geological survey and Geological survey of Nigeria. Open file report. $1968 ; 48$.

6. Matheis G. Short review of the geology of Chad Basin in Nigeria. In: Kogbe, editor. Geology of Nigeria. Elizabeth publishing Nigeria. 1976;289-294.

7. Peters SW. Stratigraphy of Chad and lullemeden Basins (West Africa). Eclogae Geol. 1981;74:139-159. 
8. Adegoke SO, Jan Du Chene RE, Agumanu AE, et al. Palynology and age of the Keri-Keri Formation, Nigeria. Revista espan Micropaleontologica. 1978;10(2):287-283.

9. Beacon Services Ltd. Consulint International. Maiduguri water supply. Hydrogeological report. Hydrogeology and future exploitation of groundwater. Consulint Int Srl. 1979;1:158.

10. Dar Al-Handasah consultants (share and partners). Maiduguri water supply project. Interim report. 1981;1:89.

11. Goni IB, Travi Y, Edmunds WM. Estimating groundwater recharge from modeling unsaturated zones using chloride profiles in the Nigerian sector of the Chad Basin. Jour Min \& Geol. 2005;41(1):123-130.

12. Oteze GE, Fayose EA. Regional development in the hydrogeology of Chad Basin. Water resources. 1988;1(1):9-29.

13. Offodile ME. Groundwater level fluctuation in the East Chad basin of Nigeria. Journ of Mining and Geol. 1972;7(2):19-34.

14. Goni IB, Kachalla M, Aji MM. Another look at the piezometric head decline in Middle zone aquifer of the Chad Formation. Borno jour of Geol. 2000;2:53-64.

15. Edmunds JW, Felman E, Goni IB. Lakes, groundwater and palaeohydrology. Jour of the Geol Soc London. 1999;156(2):345-355.

16. WHO. Fluorine and Fluorides. Environmental Health Criteria 36. World Health Organisation Geneva. 2004.

17. Vásquez V L, Ramírez-Hernández J, YeyesLópez J, et al. The origin of fluoride in groundwater supply to Hermosillo City, Sonora, Mexico. Environ Geol. 2006;51(1):17-27.
18. Goni IB. Tracing stable isotope values from meteoric water to groundwater in the southwestern part of Chad Basin. Jour Hydrog. 2006;1(5):740-751.

19. Ramakrishnan S. Groundwater. Ramakrishnan: Chennai. 1998;761.

20. Gupta SK, Deshpande RD, Agarwal M, et al. Origin of high fluoride in groundwater in the North Gujarat-Cambay region, India. Physical Research Lab., Ahmedabad, India. Hydrog J. 2004;2(13):596-605.

21. Saxena VK, Ahmed S. Dissolution of fluoride in groundwater: a water rock interaction study. Environ Geology. 2001;40(9):1084-1087.

22. Chandrasekharam D, Antu MC. Geochemistry of Tattapani thermal springs, Madhyapradesh, India field and experimental investigations. Geothermics. 1995;24(4):553-559.

23. Handa BK. Geochemistry and Genesis of fluoride containing groudwaters in India. Groundwater. 1975;13(3):275-281.

24. Bowen HJM. Trace Elements in Biochemistry. Academic Press: New York. 1966.

25. Sugawara K. Migrodun of Element through Phases of the Hydrosphere and atmosphere. In: Vingradov AP, editor. Chemistry of the earth's Crust. Israel program for Scientific Translations, Jemsalem. 1967;2:50.

26. Garg VK, Singh B. Fluoride in Drinking and fluorosis. Guru Jambheshwar: University Haryana-India. 2007;32.

27. Teotia SPS, Teotia M. Endemic Flouride. Bone and teeth update flourisis in India. Inst of Soc Sci. 1992;52-61.

28. Avbovbo AA, Ayoola EO, Osahon GA. Depositional and structural styles in Chad basin of northeastern Nigeria. AAPG Bull. 1986;70(12):17871798. 